\title{
A model for simulation and patient-specific visualization of the tissue volume of influence during brain microdialysis.
}

Elin Diczfalusy, Peter Zsigmond, Nil Dizdar, Anita Kullman, Dan Loyd and Karin Wårdell

\section{Linköping University Post Print}

N.B.: When citing this work, cite the original article.

The original publication is available at www.springerlink.com:

Elin Diczfalusy, Peter Zsigmond, Nil Dizdar, Anita Kullman, Dan Loyd and Karin Wårdell, A model for simulation and patient-specific visualization of the tissue volume of influence during brain microdialysis., 2011, Medical and Biological Engineering and Computing, (49), $12,1459-1469$.

http://dx.doi.org/10.1007/s11517-011-0841-0

Copyright: Springer Verlag (Germany) http://www.springerlink.com/

Postprint available at: Linköping University Electronic Press http://urn.kb.se/resolve?urn=urn:nbn:se:liu:diva-72911 


\title{
A model for simulation and patient-specific visualization of the tissue volume of influence during brain microdialysis
}

Elin Diczfalusy ${ }^{1}$, Peter Zsigmond ${ }^{2,3}$, Nil Dizdar ${ }^{3,4}$, Anita Kullman ${ }^{3}$, Dan Loyd ${ }^{5}$, Karin Wårdell ${ }^{1}$

${ }^{1}$ Department of Biomedical Engineering, Linköping University, Linköping, Sweden

${ }^{2}$ Department of Neurosurgery, Linköping University Hospital, Linköping, Sweden

${ }^{3}$ Department of Clinical and Experimental Medicine, Linköping University, Linköping, Sweden

${ }^{4}$ Department of Neurology, Linköping University Hospital, Linköping, Sweden

${ }^{5}$ Department of Management and Engineering, Linköping University, Linköping, Sweden

\section{Corresponding author:}

Elin Diczfalusy

Dept. of Biomedical Engineering, Linköping University

SE-581 85 Linköping

E-mail: elin.diczfalusy@liu.se

Phone: +4613286746

Total number of words of the manuscript: $\mathbf{6 6 8 1}$

Number of words of the abstract: $\mathbf{2 0 0}$

Number of figures: 6

Number of tables: 2

\begin{abstract}
Microdialysis can be used in parallel to deep brain stimulation (DBS) to relate biochemical changes to the clinical outcome. The aim of the study was to use the finite element method (FEM) to predict the tissue volume of influence $\left(\mathrm{TVI}_{\max }\right)$ and its crosssectional radius $\left(\mathrm{r}_{\mathrm{TVImax}}\right)$ when using brain microdialysis, and visualize the $\mathrm{TVI}_{\max }$ in relation to patient anatomy.

An equation based on Fick's law was used to simulate the TVI $I_{\max }$. Factorial design and regression analysis were used to investigate the impact of the diffusion coefficient, tortuosity and loss rate on the $\mathrm{r}_{\text {TVImax }}$. A calf brain tissue experiment was performed to further evaluate
\end{abstract}


these parameters. The model was implemented with pre- (MRI) and post- (CT) operative patient images for simulation of the $\mathrm{TVI}_{\max }$ for four patients undergoing microdialysis in parallel to DBS.

Using physiologically relevant parameter values, the $\mathrm{r}_{\mathrm{TVImax}}$ for analytes with a diffusion coefficient $\mathrm{D}=7.5 \cdot 10^{-6} \mathrm{~cm}^{2} / \mathrm{s}$ was estimated to $0.85 \pm 0.25 \mathrm{~mm}$. The simulations showed agreement with experimental data. Due to an implanted gold thread, the catheter positions were visible in the postoperative images. The $\mathrm{TVI}_{\max }$ was visualized for each catheter. The biochemical changes could thereby be related to their anatomical origin, facilitating interpretation of results.

Keywords: Microdialysis · Deep Brain Stimulation · Diffusion · Finite Element Method . Patient-specific visualization 


\section{Introduction}

Microdialysis is a method for monitoring of the local biochemical environment in a region of interest, and is a commonly used technique for sampling biochemical substances in the brain [1]. The method uses a catheter, mimicking a blood capillary, which can be inserted into a variety of tissues. Cerebral microdialysis is used for bedside monitoring in neuro-intensive care [2] as well as a research tool within areas such as drug delivery and neuro-oncology [3].

A recent application for brain microdialysis is the sampling of neuroactive substances in the basal ganglia during deep brain stimulation (DBS) [4-6]. Galati and colleagues [4] used microdialysis for sampling in the substantia nigra pars reticulata $(\mathrm{SNr})$ during DBS, while Stefani et al [5] investigated biochemical changes in the putamen and internal globus pallidus (GPi). At Linköping University Hospital, microdialysis has been used during DBS implantation for investigation of the pharmacokinetics of L-dopa in relation to the electric stimulation [7]. Recently, microdialysis has also been used post-operatively in parallel to DBS for patients with Parkinson's disease (unpublished material). For these patients, a large number of neuroactive substances are under investigation, to correlate electric stimulation of the subthalamic nucleus (STN) with local biochemical changes in surrounding basal ganglia nuclei.

For microdialysis measurements in general it would be highly beneficial if the sampling volume around the catheter could be predicted. This becomes especially important when performing microdialysis in the basal ganglia, since these nuclei are in the millimeter range and there are multiple biochemical pathways connecting them [8]. When analyzing microdialysis data in these structures, it is therefore desirable to estimate and visualize the position and sampling volume for each microdialysis catheter in relation to patient anatomy. In this way, the biochemical data can be interpreted in relation to anatomical targets.

Mathematical formulas and models have been developed in order to describe the movement of analytes in brain tissue [9-11]. Diffusion, as described by Fick's law, is considered the dominating process for analyte transport in tissue $[11,12]$. If analyte movement is assumed to occur in the extracellular space, a process known as volume transmission [13], diffusion in vivo can be satisfactorily described by using a volume averaged formulation of Fick's diffusion equation $[9,11]$. The input parameters of this equation have been extensively 
evaluated for different parts of the brain [10]. Furthermore, the equation has been implemented using the finite element method (FEM) and finite volume discretization methods, in order to simulate and visualize movement of analytes in the brain $[14,15]$.

In the present study we have used FEM for estimation and visualization of the volume of influence associated with microdialysis catheters positioned in basal ganglia nuclei. The overall aim was to develop a FEM model for prediction of the tissue volume from which biochemical data is obtained, and evaluate the model input parameters using statistical analysis and experimental data. A second aim was to implement the model with pre- and postoperative images for patients undergoing microdialysis in parallel to DBS, in order to structure-specifically predict the location and associated sampling volume of each microdialysis catheter.

\section{Material and Methods}

\subsection{Microdialysis FEM model}

\subsubsection{Governing equations}

By using volume averaging and assuming that the molecules are restricted to the extracellular space of the tissue, the migration of molecules in brain tissue can be described using a volume averaged form of Fick's diffusion equation $[9,10]$ :

$$
\frac{\partial \mathrm{C}}{\partial \mathrm{t}}=\frac{\mathrm{D}}{\lambda^{2}} \nabla^{2} \mathrm{C}+\frac{\mathrm{Q}}{\alpha}-\overrightarrow{\mathrm{v}} \nabla \mathrm{C}-\frac{\mathrm{f}(\mathrm{C})}{\alpha}
$$

where $C(\mathrm{nmol} / \mathrm{L})$ is the analyte concentration in the extracellular space as a function of space and time, $C(x, y, z, t) . D\left(\mathrm{~cm}^{2} / \mathrm{s}\right)$ is the analyte diffusion coefficient in solution and $\lambda$ is the tortuosity. $\lambda$ is a scalar when the tissue is isotropic, and a second-order tensor in case of anisotropy. $\alpha$ is a scalar corresponding to the volume fraction of the extracellular space. $Q / \alpha$ represents analyte generation in the tissue. The term $\vec{v} \nabla C$ stands for bulk flow, where $\vec{v}$ is a velocity vector and the velocities are functions of space and time. $f(C) / \alpha$ is the rate of analyte loss from the extracellular space. In order to solve the diffusion problem, equation (1) must be combined with suitable assumptions, boundary conditions and initial conditions. 


\subsubsection{Maximum tissue volume of influence}

It is here expected that the maximum sampling distance of a substance for a certain time interval is equal to the maximum distance that the same substance is able to diffuse in tissue during the same time interval. Under this assumption, the maximum sampling distance for an analyte during microdialysis can be predicted by simulating reverse microdialysis, i.e. release of the analyte from the catheter into the tissue. Therefore, the maximum tissue volume of influence $\left(\mathrm{TVI}_{\max }\right)$ and its associated cross-sectional radius $\left(\mathrm{r}_{\mathrm{TVImax}}\right)$ for a microdialysis fraction was defined by implementing equation (1), together with the following assumptions:

- The bulk flow term $\vec{v} \nabla C$ and the source term $Q$ are neglected.

- The loss term is set to $f(C)=k \alpha C$, where $k\left(\mathrm{~s}^{-1}\right)$ is a loss rate constant describing linear, nonspecific uptake and metabolism of the analyte.

- $\quad k, D$ and $\lambda$ are used as input parameters and set for each simulation.

- The analyte concentration at the catheter boundary is set to a constant, $c_{b}$. The outer boundary of the $\mathrm{TVI}_{\max }$ is defined with a concentration isolevel, connecting the points where $c=0.01 c_{b}$.

The resulting equation becomes:

$$
\frac{\partial \mathrm{C}}{\partial \mathrm{t}}=\frac{\mathrm{D}}{\lambda^{2}} \nabla^{2} \mathrm{C}-\mathrm{kC}
$$

In order to simulate and visualize the $\mathrm{TVI}_{\max }$, equation (2) is combined with boundary and initial conditions according to Figure 1a. The defined $\mathrm{r}_{\mathrm{TVImax}}$ and associated $\mathrm{TVI}_{\max }$ are visualized in Figure $1 b-1 d$. The $r_{T V I m a x}$ corresponds to the migration distance of the analyte molecules, with the stated isolevel, in relation to the catheter. If diffusion is isotropic, the $r_{\text {TVImax }}$ will have one single value. For anisotropic diffusion, the $r_{\text {TVImax }}$ must be further divided into $\mathrm{x}, \mathrm{y}$ and $\mathrm{z}$ components.

\subsubsection{Model input parameters}

The model was statistically evaluated by varying the following parameters: 
The diffusion coefficient, $D$ : In this study, the TVI $\mathrm{I}_{\max }$ will be evaluated for $D$ values in the range $4 \cdot 10^{-6}-1 \cdot 10^{-5}\left(\mathrm{~cm}^{2} / \mathrm{s}\right)$. This interval covers the neuroactive analytes of interest [16], as well as the analyte used for the experimental evaluation.

The loss rate constant, $k$ : The values of $k$ are assumed to be evenly distributed on the interval $0.003-0.010 \mathrm{~s}^{-1}$, based on literature data for small molecules, such as neurotransmitters, when specific, high-affinity uptake is absent [17-19].

The tortuosity value, $\lambda$ : The values of $\lambda$ are assumed to be normally distributed with a mean of 1.59 and a standard deviation of 0.096 , based on experimental values from a number of studies [10].

Anisotropy: In the current study, catheters are placed in basal ganglia nuclei which are considered to consist mainly of isotropic, grey matter. However, parts of the basal ganglia are crossed by white fiber tracts [20], and the impact of different degrees of anisotropy on tissue diffusion will therefore be evaluated and visualized. Anisotropic tortuosity values ranging from 1.4-1.95 [21-23] will act as the basis for the anisotropy evaluation.

\subsection{Model evaluation}

\subsubsection{Simulations}

Simulations (total $n=448$ ) were performed in order to evaluate the microdialysis model parameters. FEM software (Comsol Multiphysics 3.5, Comsol AB, Sweden) was used for setting up the microdialysis simulations. The catheter model geometry was based on the dimensions of the catheter used for the ex vivo and in vivo experiments (CMA64 and CMA65, CMA Microdialysis AB, Sweden). The simulations were performed using equation (2), with boundary and initial conditions according to Figure 1a. The model was solved using Comsol Multiphysics’ iterative system solver GMRES. 


\subsubsection{Statistical evaluation of input parameters}

A $2^{3}$-factorial design was used for a first rough estimation of the impact of each input parameter on the $\mathrm{r}_{\mathrm{TVImax}}$ within the parameter intervals of interest (Table 1, Table 2). Based on the factorial design, an effect plot for each parameter was created.

Regression analysis was carried out to model the simulated $\mathrm{r}_{\mathrm{TVImax}}$ and its associated prediction interval as a function of $D$, with varying values of $\lambda$ and $k$. Isotropic diffusion was assumed, and $\lambda$ was thereby considered a scalar quantity. The value of $D$ was varied in steps of $0.25 \cdot 10^{-6}$ over the stated interval, and 14 simulations were performed for each $D(n=350)$. For each simulation, a randomly generated combination of $k$ and $\lambda$ was used, based on the stated distributions. The regression analysis was performed using Minitab (Minitab Inc., USA).

To visualize how the $\mathbf{r}_{\text {TVImax }}$ was affected by different degrees of anisotropy, simulations were carried out with $\lambda$ as a second-order tensor. The simulations were performed in two dimensions, using three different tensor values of $\lambda$ with increasing degree of anisotropy. The value of $D$ was set to $4.2 \cdot 10^{-6} \mathrm{~cm}^{2} / \mathrm{s}$. Thirty simulations were performed for each $\lambda$ (total $\mathrm{n}=$ 90 ), and the values of the $\mathrm{r}_{\mathrm{TVImax}}$ in the $\mathrm{x}$ and $\mathrm{y}$ direction were measured. A randomly generated value of $k$, within the given parameter interval, was used for each simulation.

\subsubsection{Ex vivo evaluation}

In order to estimate diffusion in the deep brain structures, a microdialysis experiment was performed on brain tissue from calf obtained from the local slaughterhouse (use approved by the Swedish Board of Agriculture, D.O. 38-172/09). The microdialysis catheter (CMA64, CMA Microdialysis AB, Sweden) was placed into the basal ganglia, aiming at the putamen, by using a Venflon ${ }^{\circledR}$ catheter as guide. Crystal violet solution, with molecular weight of $407.98 \mathrm{~g} / \mathrm{mol}$ (Sigma Aldrich, St Louis, USA), was used as perfusion fluid. The flow rate was set to $0.5 \mathrm{l} / \mathrm{min}$ and microdialysis was performed for $1 \mathrm{~h}$. The tissue was cut in $1 \mathrm{~mm}$ thick slices $(n=10)$ perpendicular to the catheter membrane, and the cross-sectional radius of the coloured surface corresponding to the migration of crystal violet was measured in two directions. To improve accuracy, the experiment was repeated three times (total $n=30$ ). To 
compare the experiment to the FEM simulations and the statistical analysis, the experiment images were superimposed with traced circles corresponding to the predicted $\mathrm{r}_{\mathrm{TVImax}} . D$ for crystal violet was assumed to be $4.2 \mathrm{~cm}^{2} / \mathrm{s}$, based on manufacturer data.

\subsection{Patient-specific microdialysis FEM models}

The presented FEM model was here implemented together with image data for patients undergoing microdialysis in the basal ganglia in parallel to DBS.

\subsubsection{Patient data and surgical procedure}

Four patients (aged $56 \pm 8$ ) referred for bilateral implantation of DBS electrodes in the STN, were included in the study. In addition to the DBS electrodes, three microdialysis catheters (CMA65, CMA Microdialysis AB, Sweden) were stereotactically implanted in the putamen (right side) and the GPi (left and right side). The patients gave informed written consent for participation in the study (Ethically approved by the Regional Ethics Committee at Linköping University, No. 51-04). Stereotactic imaging (1.5 T MR-scanner, Philips Intera, The Netherlands) was performed after placement of the Leksell ${ }^{\circledR}$ Stereotactic System (model G, Elekta Instrument AB, Sweden). A stereotactic CT (GE Lightspeed Ultra, GE Healthcare, UK) was done directly after the implantations.

Following imaging, each patient was referred to the neuro-intensive care unit where collection of biochemical samples was initiated. Microdialysis samples were obtained in one-hour fractions (two-hour fractions for baseline recordings during nighttime), with a flow rate of 0.5 $\mu 1 /$ min for a total period of 60 hours. The microdialysis samples were analyzed with regard to several neuroactive substances (including dopamine, GABA, glutamate and serotonin) using high pressure liquid chromatography (HPLC) with electrochemical detection. The microdialysis monitoring is part of an ongoing study, and the results will be presented in a separate paper when finished.

\subsubsection{Brain and catheter models}

Three-dimensional FEM models of the microdialysis catheters were created based on their real dimensions. A software tool developed in MatLab 7.6 (The MathWorks, USA) was used 
to set up tissue intensity and property matrices based on the pre- and postoperative patient images, as previously presented by Åström et al [24]. An overview of the simulation process is shown in Figure 2. Three property matrices were used to set up each patient-specific model:

Matrix 1: The postoperative CT images were used to create an intensity matrix for catheter placement. A gold marker in the tip of each catheter was visible as an artefact in the CT images and acted as reference positions for the catheter models.

Matrix 2: The preoperative MRI images were used to create an intensity matrix, to act as reference for the visualization of patient anatomy. The matrix included a tissue volume corresponding to the basal ganglia and part of the midbrain. Each voxel was represented with an intensity value, corresponding to that of the original MRI image.

Matrix 3: A property matrix with the same size and resolution as Matrix 2 was created, where each voxel value was replaced with a scalar value representing the effective diffusion coefficient, $\mathrm{D}_{\mathrm{e}}=\mathrm{D} / \lambda^{2}$.

\subsubsection{Simulations and visualization}

For each patient, the $\mathrm{TVI}_{\max }$ around each catheter model was simulated by using equation (2), using $D=7.5 \cdot 10^{-6} \mathrm{~cm}^{2} / \mathrm{s}$ and the other parameters at their mean values $(\lambda=1.59, k=0.0065$ $\mathrm{s}^{-1}$ ). FEM simulation software (COMSOL Multiphysics 3.5, Comsol AB, Stockholm, Sweden) was used, with boundary and initial conditions according to Figure 1. The simulations were performed in a three-dimensional Cartesian co-ordinate system using the iterative system solver GMRES. The outline of the $\mathrm{TVI}_{\max }$ for each catheter was superimposed onto the preoperative intensity images. Anatomical structures as extracted from the Morel and Schaltenbrandt brain atlases $[25,26]$ were traced onto the images.

\section{Results}

The evaluation of the microdialysis FEM model is presented with data from the $2^{3}$-factorial design and the regression analysis, and with images where the modelled $\mathrm{r}_{\mathrm{TVImax}}$ is related to 
the ex vivo microdialysis experiment. The simulations and visualizations of the $\mathrm{TVI}_{\max }$ are presented in relation to patient anatomy.

\subsection{Microdialysis FEM model}

\subsubsection{Statistical evaluation of input parameters}

Figure 3a shows an effect plot, predicting the estimated effect of each input parameter $(D, k$, $\lambda$ ) on the simulated $\mathrm{r}_{\mathrm{TVImax}}$ based on the $2^{3}$-factorial design. The plot indicates that an increasing value of $D$ increases the $\mathrm{r}_{\mathrm{TVImax}}$, while increasing values of $\lambda$ and $k$ decreases it. Within the current parameter intervals, an increasing value of $k$ has a larger effect on the $\mathrm{r}_{\mathrm{TVImax}}$ than an increase in $\lambda$.

Figure $3 \mathrm{~b}$ presents the results of the regression analysis, including the fitted regression line and an associated double-sided $95 \%$ prediction interval. When studying the residual histogram for the simulation results, a tendency towards larger variations for large values of $D$ was seen. Therefore, logarithm transformation was applied to the values of the response variable ( $\left.\mathrm{r}_{\mathrm{TVImax}}\right)$ before the analysis was carried out. The following regression model was obtained:

$$
\log (r)=-0.429+0.693 D-0.279 D^{2}
$$

where $\mathrm{r}$ corresponds to the $\mathrm{r}_{\mathrm{TVImax}}(\mathrm{mm}) \cdot R^{2}=41.1 \%(\mathrm{p}<0.05)$, showing that there is a large variability that cannot be explained by the model. The model shows that an increased value of $D$ increases the $\mathrm{r}_{\mathrm{TVImax}}$, which is in accordance with the results from the factorial design. For analytes with $D$ values within the interval of interest, the $\mathrm{r}_{\mathrm{TVImax}}$ ranges from $0.6-0.9 \mathrm{~mm}$. For a neurotransmitter with $D=7.5 \cdot 10^{-6} \mathrm{~cm}^{2} / \mathrm{s}$, the $\mathrm{r}_{\mathrm{TVImax}}$ is $0.85 \mathrm{~mm}$ with a $95 \%$ prediction interval ranging over about $0.5 \mathrm{~mm}$.

\subsubsection{Ex vivo microdialysis}

Two representative images from the ex vivo microdialysis experiment are shown in Figure 4, superimposed with traced circles corresponding to the axial extension of the modelled $\mathbf{r}_{\mathrm{TVImax}}$. The tissue slice images from the ex vivo experiment showed a mean cross-sectional radius, 
corresponding to the migration of crystal violet, of $0.64 \pm 0.08 \mathrm{~mm}$. This corresponds well to the regression model prediction for crystal violet (Equation (3), using $D=4.2 \cdot 10^{-6} \mathrm{~cm}^{2} / \mathrm{s}$ ), which gives $r_{\mathrm{TVImax}}=0.65 \mathrm{~mm}$ with an associated prediction interval ranging from 0.48 to $0.86 \mathrm{~mm}$.

In Figure 4(a), each image has been superimposed with a circle corresponding to the $\mathrm{r}_{\mathrm{TVImax}}$ for crystal violet according to the regression model (Equation (3)). The associated 95\% prediction interval of the regression model is included. For the right image, there is overall agreement between the coloured surface and the model prediction. For the left image, the $\mathrm{r}_{\mathrm{TVImax}}$ is well fitted to the outer boundary of the coloured surface in one direction and clearly overestimated in the other. In Figure 4(b) - 4(d), the same images are superimposed with circles corresponding to the $r_{x}$ and $r_{y}$ when simulating anisotropic diffusion, using the mean value and standard deviation for each set of simulations. It is seen that an anisotropic model fits the borders of the left images considerably better than the isotropic regression model, and that anisotropic diffusion affects the shape of the TVI $\max$.

\subsection{Patient-specific microdialysis FEM models}

Figure 2 shows that due to the artefacts originating from the gold tips, the position and direction of each microdialysis catheter can be confirmed in relation to patient anatomy. Thereby, the postoperative images provide a suitable basis when localizing the catheter positions for the microdialysis simulations. Figure 5 presents the simulation of the $\mathrm{TVI}_{\max }$ in relation to anatomy for each patient, for a substance with $\mathrm{D}=7.5 \cdot 10^{-6} \mathrm{~cm}^{2} / \mathrm{s}$. The simulated $\mathrm{r}_{\mathrm{TVImax}}$ for each catheter is $0.85 \mathrm{~mm}$ in each direction, as predicted from Equation (3). In Figure 5a, three-dimensional images are used to provide a spatial overview of the $\mathrm{TVI}_{\max }$ in relation to patient anatomy. In Figure 5b, axial MRI slices are shown, including outlines of the modelled $\mathrm{TVI}_{\max }$ and the extracted brain atlas contours $[25,26]$. The upper limit of the 95\% prediction interval for the $r_{\mathrm{TVImax}}$ for each catheter, according to Equation (3), has been added. It is seen that the $\mathrm{r}_{\mathrm{TVImax}}$ is sufficiently small to relate microdialysis data to one main nucleus for each catheter, and that there are differences regarding target areas between the patients. For patient 1 and 3, the catheters and their associated sampling volumes are located within the anatomical targets aimed at. For patient 2, the right globus pallidus externa (GPe) is sampled rather than the right GPi. For patient 4, two catheters and their associated TVI max $_{\text {can }}$ be traced to the putamen, and the third to the substantia nigra. 


\subsection{Microdialysis data}

In order to demonstrate how the model can be used clinically, microdialysis data for dopamine $\left(\mathrm{D}=7.5 \cdot 10^{-6} \mathrm{~cm}^{2} / \mathrm{s}[16]\right)$ for patient 1 is shown in Figure 6. The data is presented together with the DBS stimulation protocol, indicating that this protocol is suitable for studying changes of transmitter levels in the basal ganglia. As presented for Patient 1 in Figure 5, the simulated $\mathrm{TVI}_{\max }$ limits the microdialysis data for each catheter to one main anatomical target area. Following stimulation, a dopamine concentration increase can be observed for one catheter only, thereby indicating that there are differences in the biochemical signaling between the sampled targets.

\section{Discussion}

The present study demonstrates how analyte diffusion simulations can be combined with post-operative patient images, in order to visualize the maximum tissue volume that is being sampled during brain microdialysis. In this way, the microdialysis data to be analyzed can be related to their anatomical origin. Figure 6 confirms that changes in neurotransmitter levels clearly differ between catheters placed in different anatomical targets, thereby verifying the need to confirm the target area for each catheter.

The defined $\mathrm{TVI}_{\max }$ is a qualitative estimation of the maximum tissue volume that can be sampled during brain microdialysis. For a substance with $\mathrm{D}=7.5 \cdot 10^{-6} \mathrm{~cm}^{2} / \mathrm{s}$, representative for that of several neuroactive substances [16], the $\mathrm{r}_{\mathrm{TVImax}}$ can be predicted with an accuracy of $0.85 \pm 0.25 \mathrm{~mm}$. As can be seen in Figure 5, most basal ganglia nuclei are a few millimeters in size, and the defined $\mathrm{TVI}_{\max }$ can in most cases be traced to one anatomical target. In such cases, the $\mathrm{TVI}_{\max }$ confirms which target is being sampled and excludes the involvement of neighbouring structures. It is therefore clinically beneficial to visualize the $\mathrm{r}_{\mathrm{TVImax}}$ and associated $\mathrm{TVI}_{\max }$ for a catheter when using brain microdialysis, to determine whether the measurement sites are correct and comparable between patients when interpreting physiological data. The extension of the $\mathrm{TVI}_{\max }$ is also reasonable in comparison to previous studies, predicting that the microdialysis penetration depth for similar substances would be within the millimeter range [27-29]. 


\subsection{Model assumptions and limitations}

The idea of the $\mathrm{TVI}_{\max }$ is to simulate the maximum analyte migration distance $\left(\mathrm{r}_{\mathrm{TVImax}}\right)$ in all directions from the microdialysis catheter, using physiologically relevant parameter values. Thereby, it provides a qualitative measurement of the maximum tissue volume that can be sampled during brain microdialysis. This information is especially valuable when the defined $\mathrm{TVI}_{\max }$ fits within one anatomical target (e.g. GPi or putamen), as for patient 1 and patient 3 in Figure 5. In such cases, it can be concluded that the biochemical data originates from that specific region, thereby excluding the influence of surrounding anatomical structures. For cases where the microdialysis catheter is located between target areas, as can be seen for patient 4 in Figure 5, the $\mathrm{TVI}_{\max }$ can be used to confirm that both areas are possibly influencing the resulting data. In order to draw specific conclusions regarding the impact of each nuclei, local tissue properties as well as the behaviour of the substance of interest in the particular tissue volume would have to be more closely evaluated.

The $\mathrm{TVI}_{\max }$ is defined and simulated by using physiologically relevant values of $D, \lambda$ and $k$ as input parameters. Similar input parameters have been used by Bungay et al [27] in order to mathematically define the spatial resolution of microdialysis. For DOPAC, a neuroactive substance with $D=7.5 \cdot 10^{-6} \mathrm{~cm}^{2} / \mathrm{s}$ [16], a $90 \%$ penetration depth of $0.35 \mathrm{~mm}$ was found in their study. The $\mathrm{r}_{\mathrm{TVImax}}$ for a substance such as DOPAC, using our approach, would be 0.85 $\mathrm{mm}$. However, if an isolevel of $90 \%$ was used instead of $99 \%$, the simulated $\mathbf{r}_{\mathrm{TVImax}}$ would be about $0.4 \mathrm{~mm}$, thereby showing good agreement with the work of Bungay et al [27]. The choice of isolevel at $99 \%$ in our study was due to the fact that the neurotransmitter concentrations, according to the obtained microdialysis data, were generally in the nanomolar range while the detection limit for the HPLC system is in the picomolar range. A biochemical change in the nanomolar range, at a distance from the probe corresponding to $\mathrm{r}_{\mathrm{TVImax}}$ at a $99 \%$ isolevel, would therefore be qualitatively detectable using the current equipment.

In this study, the microdialysis catheter locations are patient-specifically defined and visualized while the diffusion simulations are performed in a more general manner. The intension of the study was to develop a diffusion model for basal ganglia nuclei, considered to consist mainly of grey matter, and isotropic diffusion has therefore been assumed. This is valid as long as the catheter is placed in the intended target areas, since grey matter, in comparison to white matter, is considered to be isotropic [20]. As can be seen in Figure 5, 
however, the catheters may end up outside the nuclei of interest, resulting in possible anisotropic diffusion which affects the shape of the $\mathrm{TVI}_{\max }$ (Figure 4). Therefore, in order to make the models truly patient-specific, local tissue properties would have to be defined and included. Diffusion tensor imaging (DTI) has previously been used for predicting heterogenous and anisotropic drug transport in the brain [15], and could be suitable for patient-specific diffusion simulations. DTI images have low resolution, however, and may not accurately represent local tissue properties within the millimeter range.

\subsection{Impact of different parameters on the predicted $\mathrm{TVI}_{\max }$}

The regression model was created for presenting the $\mathrm{r}_{\mathrm{TVImax}}$ as a function of $D$, with the underlying assumption that the value of $D$ for an analyte can be estimated if its physical properties are known [30,31]. The values of $\lambda$ and $k$ cannot be measured for an individual patient, and the regression model was therefore based on the assumption that these parameters vary within stated intervals. Within these intervals, the variability of the $\mathrm{r}_{\mathrm{TVImax}}$ is large (about $50 \%$ ). This is reasonable with regard to the stated parameter intervals, and in accordance with previous studies [27, 32]. Even when considering the outer limit of the $95 \%$ prediction interval (Figure 5), the $\mathrm{r}_{\mathrm{TVImax}}$ is generally small enough to draw conclusions in relation to the anatomical structures of interest.

The value of $\lambda$ was assumed to be normally distributed with a mean of 1.59 and a standard deviation $<0.01$, estimated from a number of literature values [10]. A mean $\lambda$ of about 1.6 is in agreement with other studies $[9,33]$, so the assumption is considered appropriate. The $k$ values used for this study are based on data proposed for small molecules, including neurotransmitters, when high-affinity uptake is absent [10,17-19], and result in an interval causing large variations of the $\mathrm{r}_{\mathrm{TVImax}}$ (Figure 3). This is in accordance with previous studies, proposing that the variation of $k$ tend to cause a large variation of the penetration depth [27, 32]. The loss rate term takes only nonspecific, linear uptake into account, in order to keep the model as general as possible. Biologically active molecules such as neurotransmitters may experience a more complex, increased loss pattern due to specific uptake and metabolism [9, $34,35]$, which is an important factor in determining the analyte time course in certain regions of the brain $[19,36,37]$. Consequently, neglecting the specific uptake may result in a large overestimation of the $\mathbf{r}_{\text {TVImax }}$. An additional loss term for a certain substance in a particular target area could be included in future simulations in order to refine the tissue volume of 
influence. This may be useful in cases where the $\mathrm{TVI}_{\max }$ is placed between structures, as for patient 4 in Figure 5, or for applications where a more detailed estimation of the tissue volume of influence for a particular substance is needed.

Figure 4 compares the ex vivo microdialysis data to the modelled $\mathrm{r}_{\mathrm{TVImax}}$, in order to evaluate the diffusion model. Practical difficulties were experienced when handling and cutting the fresh brain tissue, which may have altered the thickness and shape of the tissue slices. However, general agreement was seen between the slices regarding shape and size of the coloured surface, suggesting that the model assumptions are reasonable. For the left image, the $\mathrm{r}_{\mathrm{TVImax}}$ is overestimated in one direction, indicating possible tissue anisotropy. When introducing different levels of anisotropy (Figure $4 b-4 d$ ), a gradually increasing agreement is seen between the image and the model data. The resulting $\mathbf{r}_{\mathrm{TVImax}}$ is, however, generally altered by less than $0.1 \mathrm{~mm}$ compared to the isotropic case. It should also be noted that the uneven shape may result from "squeezing" of the tissue during the cutting.

\subsection{Relating the model to patient data and DBS}

The second aim of the current study was to implement the microdialysis model together with patient data, in order to relate the simulated $\mathrm{TVI}_{\max }$ to anatomical targets for each patient. As can be seen in Figure 2, the postoperative CT scans can be used for localization of the microdialysis catheters due to the gold tip, thereby allowing microdialysis simulations and visualization in relation to patient anatomy. The particular application of interest for the patients included in the study is relating microdialysis data to the outcome of DBS. An example of the resulting data has been included, in order to verify that the protocol is suitable for the current application and that there is a need to differentiate between different anatomical targets. The remaining biochemical data will be presented in a future study, where the developed models will be used for prediction of the TVI $\mathrm{max}_{\max }$ for each microdialysis catheter.

In this study, FEM has been used for the microdialysis simulations. When used with suitable software, FEM is a powerful and flexible method which allows coupling of different physical phenomena. FEM has previously been used by our group for patient-specific simulations of the distribution of electric parameters around DBS electrodes [24]. For patients undergoing microdialysis in parallel to DBS, as in this study, a possible next step is to combine the 
microdialysis model with the electric field predictions to draw further conclusions about the mechanisms of DBS. The current microdialysis model can also be further developed, allowing model refinement as well as adaptation to other tissues and applications. Prediction of the volume of influence during neuro-intensive care and estimation of tissue parameters when using microdialysis in skin are two examples of clinical applications where the developed model can be used.

\section{Acknowledgments}

The authors would like to thank Johan Richter, MD, and the staff at Neurosurgical Department of Linköping University Hospital for skillful help during the surgical procedure. The authors would also like to thank Mattias Åström, PhD. and Johannes Johansson, $\mathrm{PhD}$ at the Department of Biomedical Engineering, for help with the simulations, Pontus Lindblom, MSc at the Department of Clinical and Experimental Medicine, for assistance with microscope software, and associate professor Eva Enqvist, Department of Mathematics, Linköping University, for valuable aid with the statistical design and analysis. The study was supported by the Swedish Governmental Agency for Innovation Systems (Vinnova), Swedish Foundation for Strategic Research (SSF), Swedish Research Council (VR) (Group grant no. 311-2006-7661), Research Foundation of the County Council of Östergötland, Medical Research Council of Southeast Sweden and Linköping University's Foundation for Parkinson's Research.

\section{References}

1. Chefer, V.I., A.C. Thompson, A. Zapata, and T.S. Shippenberg, Overview of brain microdialysis. Curr Protoc Neurosci, 2009. Chapter 7: p. Unit7 1.

2. Hillman, J., O. Aneman, M. Persson, C. Andersson, C. Dabrosin, and P. Mellergard, Variations in the response of interleukins in neurosurgical intensive care patients monitored using intracerebral microdialysis. Journal of neurosurgery, 2007. 106(5): p. 820-5.

3. Tisdall, M.M. and M. Smith, Cerebral microdialysis: research technique or clinical tool. British journal of anaesthesia, 2006. 97(1): p. 18-25.

4. Galati, S., P. Mazzone, E. Fedele, A. Pisani, A. Peppe, M. Pierantozzi, L. Brusa, D. Tropepi, V. Moschella, M. Raiteri, et al., Biochemical and electrophysiological changes of substantia nigra pars reticulata driven by subthalamic stimulation in patients with Parkinson's disease. Eur J Neurosci, 2006. 23(11): p. 2923-8.

5. Stefani, A., E. Fedele, S. Galati, M. Raiteri, O. Pepicelli, L. Brusa, M. Pierantozzi, A. Peppe, A. Pisani, G. Gattoni, et al., Deep brain stimulation in Parkinson's disease patients: biochemical evidence. J Neural Transm Suppl, 2006(70): p. 401-8.

6. Kilpatrick, M., E. Church, S. Danish, M. Stiefel, J. Jaggi, C. Halpern, M. Kerr, E. Maloney, M. Robinson, I. Lucki, et al., Intracerebral microdialysis during deep brain stimulation surgery. Journal of neuroscience methods, 2010. 190(1): p. 106-11.

7. Zsigmond, P., Nezirevic, D., Kullman, A., Richter, J., Augustinsson, L-E., Dizdar, N., Stereotactic Microdialysis of the Basal Ganglia in Parkinson's Disease. Submitted, 2011.

8. DeLong, M.R. and T. Wichmann, Circuits and circuit disorders of the basal ganglia. Archives of neurology, 2007. 64(1): p. 20-4.

9. Nicholson, C., Diffusion and related transport mechanisms in brain tissue. Reports on Progress in Physics, 2001. 64: p. 815. 
10. Sykova, E. and C. Nicholson, Diffusion in brain extracellular space. Physiol Rev, 2008. 88(4): p. 1277340.

11. Nicholson, C. and J.M. Phillips, Ion diffusion modified by tortuosity and volume fraction in the extracellular microenvironment of the rat cerebellum. J Physiol, 1981. 321: p. 225-57.

12. Amberg, G. and N. Lindefors, Intracerebral microdialysis: II. Mathematical studies of diffusion kinetics. J Pharmacol Methods, 1989. 22(3): p. 157-83.

13. Fuxe, K. and L. Agnati, Volume transmission in the brain : novel mechanisms for neural transmission 1991, New York: Raven Press.

14. Kalyanasundaram, S., V.D. Calhoun, and K.W. Leong, A finite element model for predicting the distribution of drugs delivered intracranially to the brain. American Journal of Physiology Regulatory, Integrative and Comparative Physiology, 1997. 273(5): p. R1810-R1821.

15. Linninger, A.A., M.R. Somayaji, T. Erickson, X. Guo, and R.D. Penn, Computational methods for predicting drug transport in anisotropic and heterogeneous brain tissue. Journal of biomechanics, 2008. 41(10): p. 2176-87.

16. Rice, M.E., G.A. Gerhardt, P.M. Hierl, G. Nagy, and R.N. Adams, Diffusion coefficients of neurotransmitters and their metabolites in brain extracellular fluid space. Neuroscience, 1985. 15(3): p. 891-902.

17. Rice, M.E. and C. Nicholson, Diffusion characteristics and extracellular volume fraction during normoxia and hypoxia in slices of rat neostriatum. J Neurophysiol, 1991. 65(2): p. 264-72.

18. Nicholson, C. and M.E. Rice, The migration of substances in the neuronal microenvironment. Ann N Y Acad Sci, 1986. 481: p. 55-71.

19. Cragg, S.J., C. Nicholson, J. Kume-Kick, L. Tao, and M.E. Rice, Dopamine-mediated volume transmission in midbrain is regulated by distinct extracellular geometry and uptake. J Neurophysiol, 2001. 85(4): p. 1761-71.

20. Mamata, H., F.A. Jolesz, and S.E. Maier, Characterization of central nervous system structures by magnetic resonance diffusion anisotropy. Neurochemistry international, 2004. 45(4): p. 553-60.

21. Mazel, T., Z. Simonova, and E. Sykova, Diffusion heterogeneity and anisotropy in rat hippocampus. Neuroreport, 1998. 9(7): p. 1299-304.

22. Rice, M.E., Y.C. Okada, and C. Nicholson, Anisotropic and heterogeneous diffusion in the turtle cerebellum: implications for volume transmission. Journal of neurophysiology, 1993. 70(5): p. 2035-44.

23. Sykova, E., T. Mazel, and Z. Simonova, Diffusion constraints and neuron-glia interaction during aging. Experimental gerontology, 1998. 33(7-8): p. 837-51.

24. Astrom, M., L.U. Zrinzo, S. Tisch, E. Tripoliti, M.I. Hariz, and K. Wårdell, Method for patient-specific finite element modeling and simulation of deep brain stimulation. Med Biol Eng Comput, 2009. 47(1): p. 21-8.

25. Morel, A., Stereotactic atlas of the human thalamus and basal ganglia2007: Informa Healthcare USA Inc.

26. Schaltenbrand, G., W. Wahren, and R.G. Hassler, Atlas for stereotaxy of the human brain 1977: Year Book Medical Publishers.

27. Bungay, P.M., P.F. Morrison, and R.L. Dedrick, Steady-state theory for quantitative microdialysis of solutes and water in vivo and in vitro. Life Sci, 1990. 46(2): p. 105-19.

28. Dykstra, K.H., J.K. Hsiao, P.F. Morrison, P.M. Bungay, I.N. Mefford, M.M. Scully, and R.L. Dedrick, Quantitative examination of tissue concentration profiles associated with microdialysis. Journal of neurochemistry, 1992. 58(3): p. 931-40.

29. Bungay, P.M., R.K. Sumbria, and U. Bickel, Unifying the mathematical modeling of in vivo and in vitro microdialysis. Journal of pharmaceutical and biomedical analysis, 2011. 55(1): p. 54-63.

30. Wilke, C.R. and P. Chang, Correlation of diffusion coefficients in dilute solutions. AIChE Journal, 1955. 1(2): p. 264-270.

31. Reddy, K.A. and L.K. Doraiswamy, Estimating Liquid Diffusivity. Industrial \& Engineering Chemistry Fundamentals, 1967. 6(1): p. 77-79.

32. Chen, K.C., M. Hoistad, J. Kehr, K. Fuxe, and C. Nicholson, Quantitative dual-probe microdialysis: mathematical model and analysis. Journal of neurochemistry, 2002. 81(1): p. 94-107.

33. Nicholson, C. and E. Sykova, Extracellular space structure revealed by diffusion analysis. Trends Neurosci, 1998. 21(5): p. 207-15.

34. Nicholson, C., Interaction between diffusion and Michaelis-Menten uptake of dopamine after iontophoresis in striatum. Biophys J, 1995. 68(5): p. 1699-715.

35. Sykova, E. and L. Vargova, Extrasynaptic transmission and the diffusion parameters of the extracellular space. Neurochemistry international, 2008. 52(1-2): p. 5-13.

36. Cragg, S.J. and M.E. Rice, DAncing past the DAT at a DA synapse. Trends Neurosci, 2004. 27(5): p. 270-7. 
37. Westerink, B.H. and J.B. De Vries, A method to evaluate the diffusion rate of drugs from a microdialysis probe through brain tissue. J Neurosci Methods, 2001. 109(1): p. 53-8.

\section{Tables}

Table 1. High and low level parameter values for the $2^{3}$-factorial design.

\begin{tabular}{lccc}
\hline & $\mathbf{D}\left[\mathbf{c m}^{2} / \mathbf{s}\right]$ & $\boldsymbol{\lambda}$ & $\mathbf{k}\left[\mathbf{s}^{-1}\right]$ \\
\hline High level (+1) & $1 \mathrm{e}^{-5}$ & 1.8 & 0.01 \\
Low level (-1) & $5 \mathrm{e}^{-6}$ & 1.4 & 0.003 \\
\hline
\end{tabular}

Table 2. Overview of the simulations for the factorial design. The resulting effects are visualized in Figure 3.

\begin{tabular}{lcccc}
\hline Simulation & $\mathbf{D}$ & $\boldsymbol{\lambda}$ & $\mathbf{k}$ & $\mathbf{r}_{\text {TVImax }}[\mathbf{m m}]$ \\
\hline 1 & -1 & -1 & -1 & 0.8296 \\
2 & -1 & -1 & +1 & 0.6417 \\
3 & -1 & +1 & -1 & 0.807 \\
4 & -1 & +1 & +1 & 0.5101 \\
5 & +1 & -1 & -1 & 1.507 \\
6 & +1 & -1 & +1 & 0.8067 \\
7 & +1 & +1 & -1 & 1.198 \\
8 & +1 & +1 & +1 & 0.6799 \\
$\sum+$ & 4.1916 & 3.195 & 2.6384 & \\
$\sum-$ & 2.7884 & 3.785 & 4.3416 & \\
$\sum+/ \mathrm{n}+$ & 1.0479 & 0.79875 & 0.6596 & \\
$\sum-/ \mathrm{n}-$ & 0.6971 & 0.94625 & 1.0854 & \\
Effect & 0.3508 & -0.1475 & -0.4258 & \\
\hline
\end{tabular}




\section{Figures}

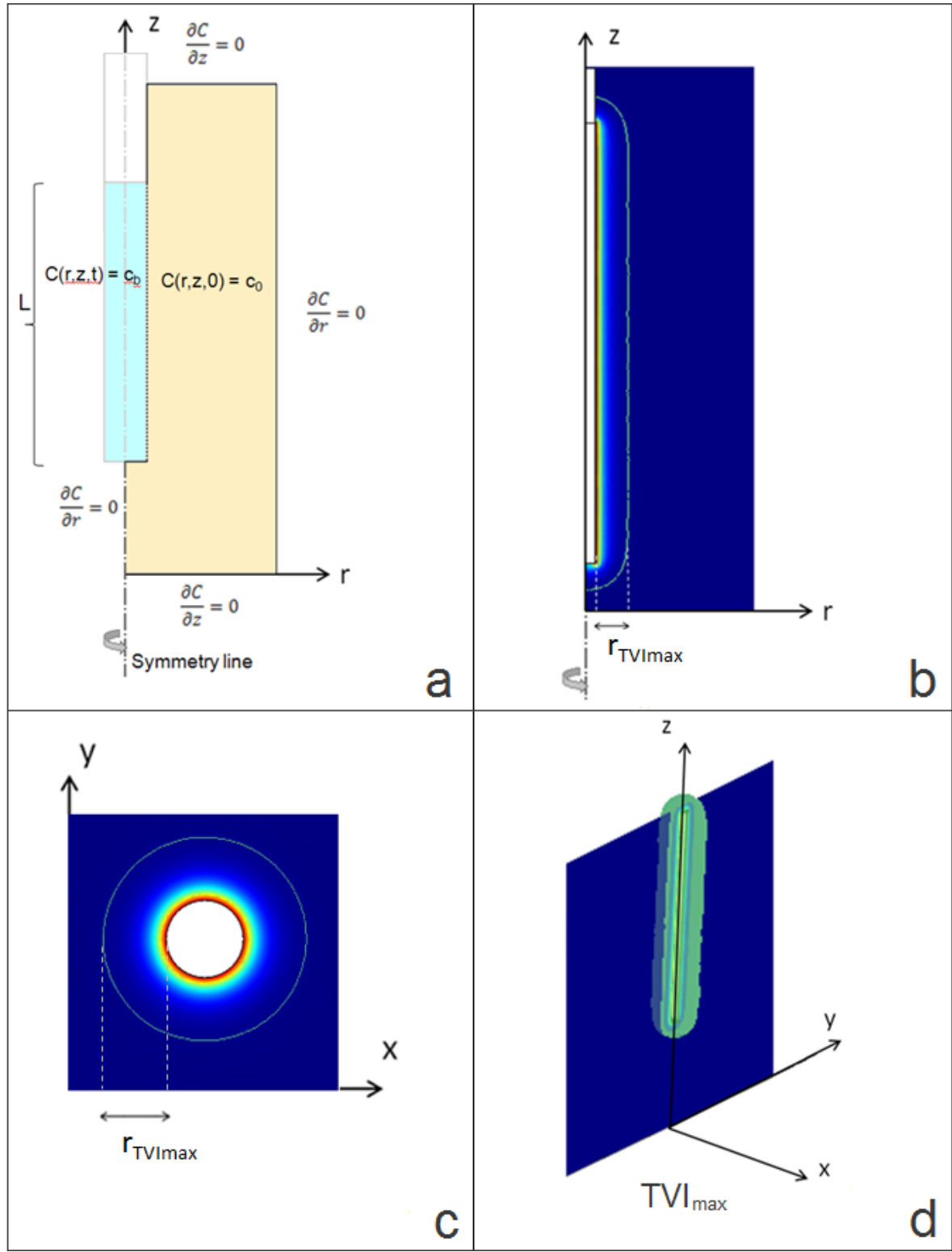

Fig. 1 (a) Boundary conditions (in italic) and initial conditions for the simulation of the $r_{\mathrm{TVImax}}$ (axi-symmetrical model). $\mathrm{L}$ is the membrane length $(\mathrm{L}=10 \mathrm{~mm}), \mathrm{c}_{\mathrm{b}}$ is the catheter boundary analyte concentration and $\mathrm{c}_{0}$ is the initial analyte concentration in the tissue. (b-c) The resulting $\mathrm{r}_{\mathrm{TVImax}}$, illustrated in an axi-symmetric co-ordinate system and in the $\mathrm{x}-\mathrm{y}$ plane $(\mathrm{d})$ The resulting $\mathrm{TVI}_{\max }$, illustrated in a three-dimensional Cartesian co-ordinate system. 


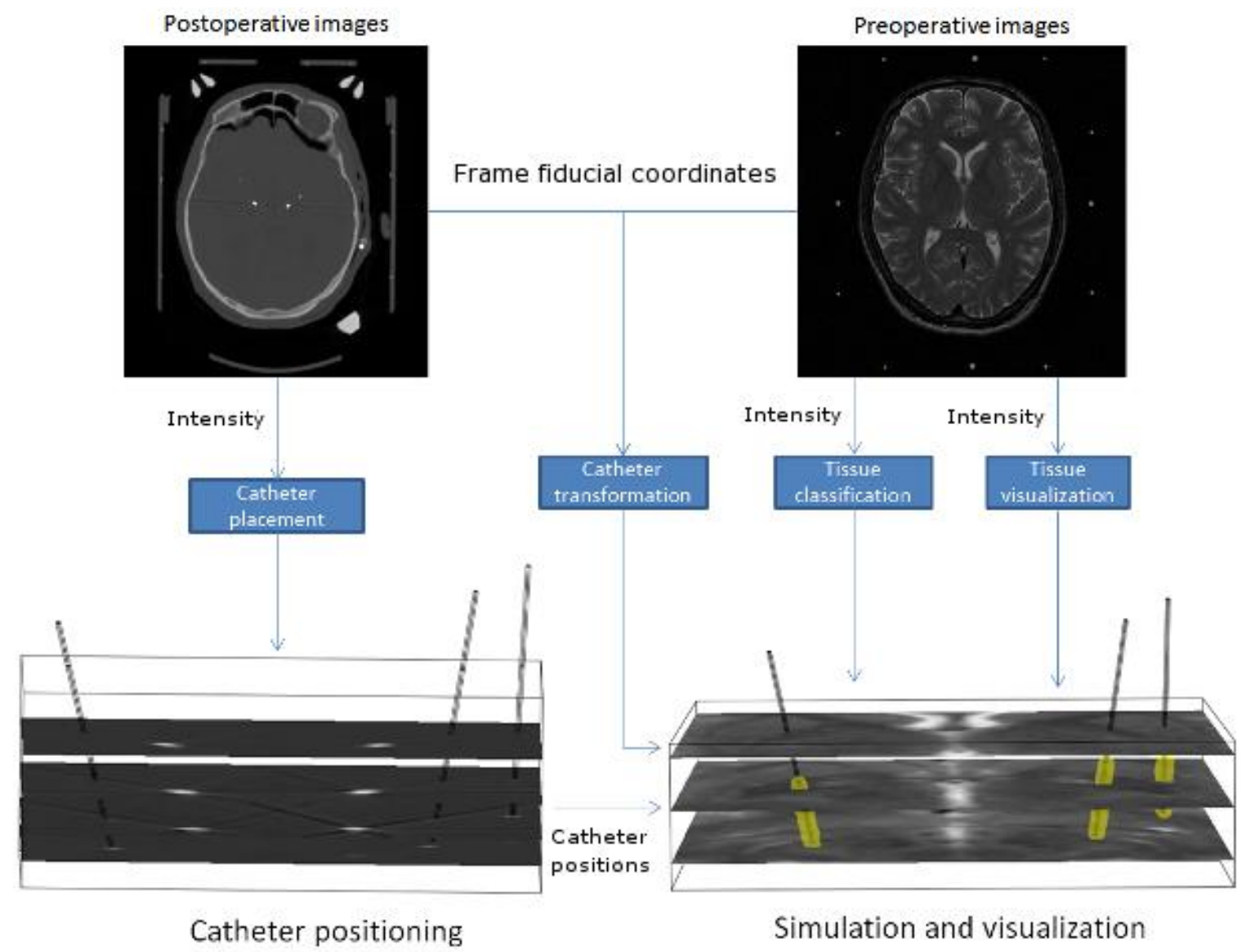

Fig. 2 The overall FEM simulation process. The gold threads in the microdialysis catheter tips are visible as artefacts in the postoperative images (the larger artefacts originate from the DBS electrodes). The postoperative images are used for positioning of the catheters, while the preoperative images are used for assignment of physical properties and tissue visualization. The frame fiducial co-ordinates are used to transfer the catheter positions to the preoperative image set. 

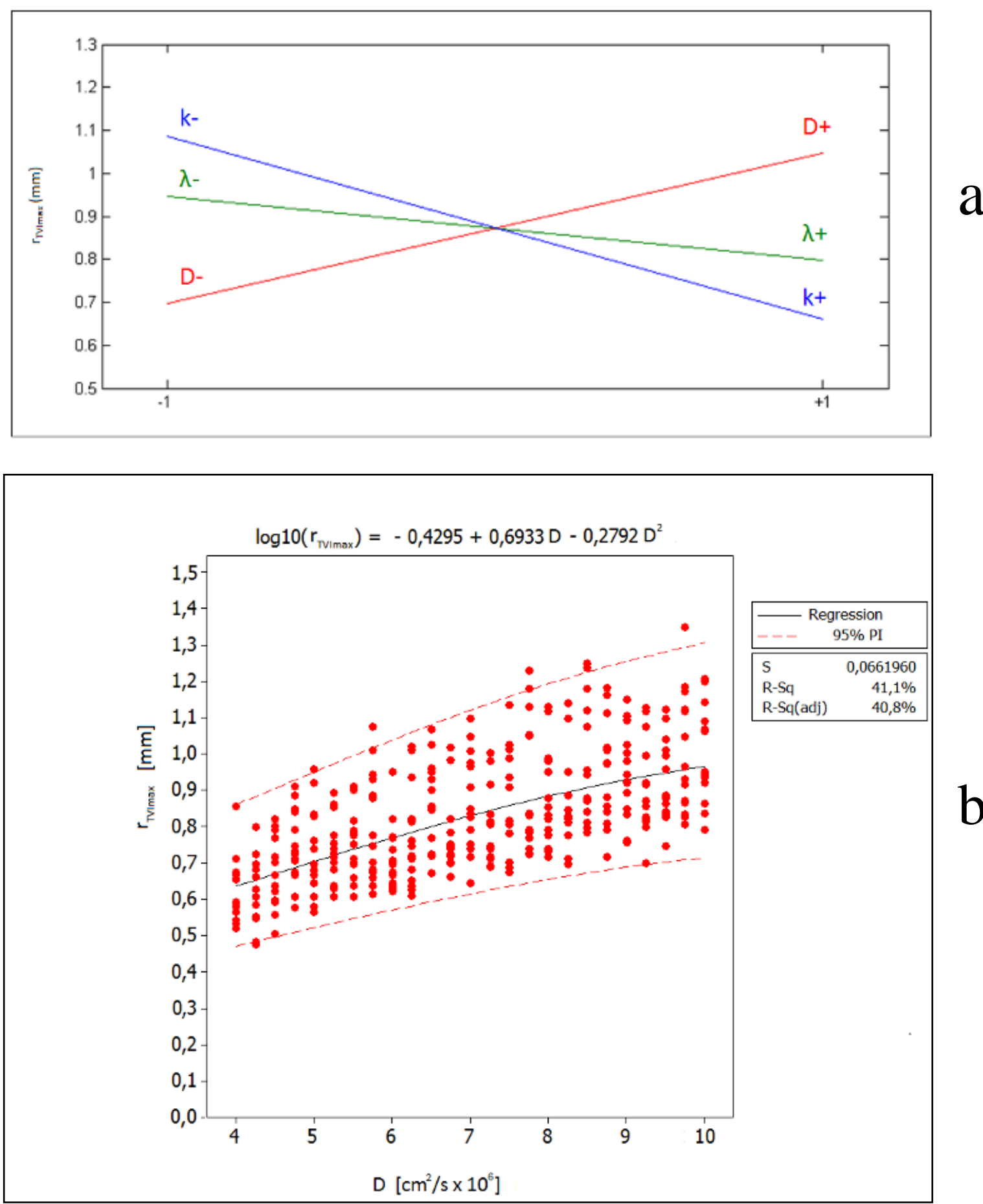

b

Fig. 3 Statistical analysis of the diffusion model. (a) Effect plot to illustrate the effect of each input parameter $(D, \lambda, k)$ on the defined $\mathrm{r}_{\mathrm{TVImax}}$, within the parameter intervals of interest. An increasing value of $D$ increases the $\mathrm{r}_{\mathrm{TVImax}}$, while an increasing value of $\lambda$ and $k$ decreases it. (b) Regression analysis based on diffusion FEM simulations $(n=350)$, where the simulated $\mathrm{r}_{\mathrm{TVImax}}$ is plotted as a function of the diffusion coefficient $(D)$ with varying values of the tortuosity $(\lambda \sim \mathrm{N}(1.59,0.096))$ and loss rate constant $(k \sim \operatorname{Re}(0.003,0.01))$. The fitted regression model is marked with a black line, and the limits of the $95 \%$ prediction interval with red lines. 


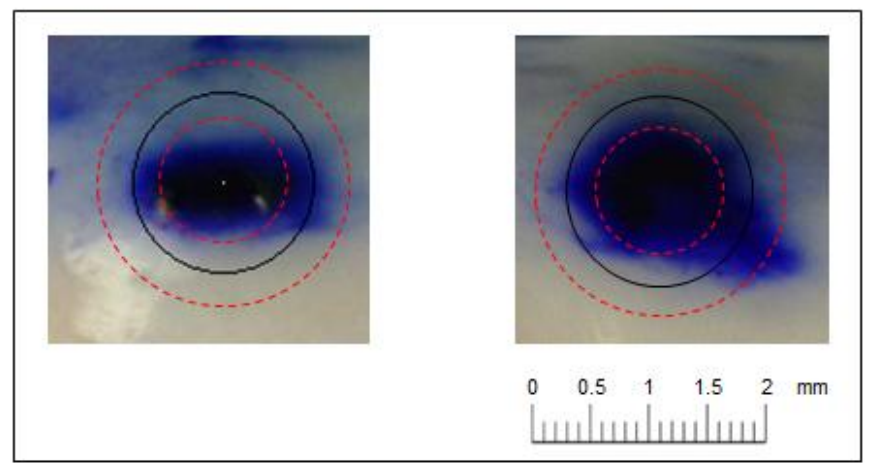

a

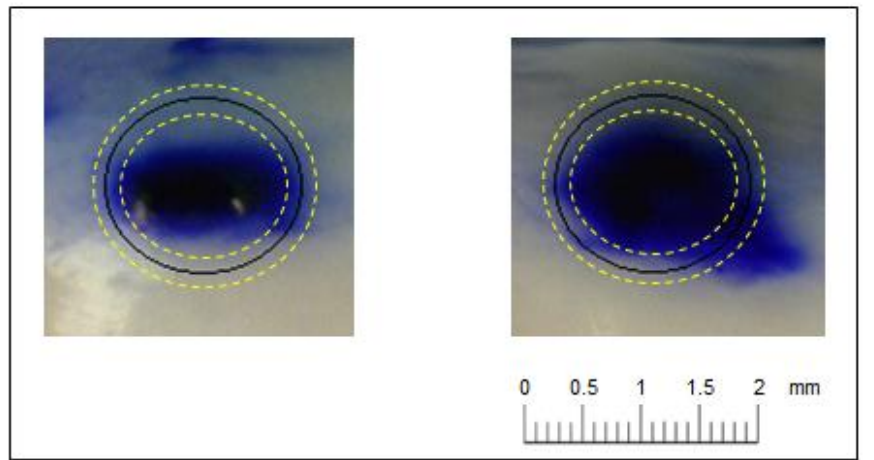

b

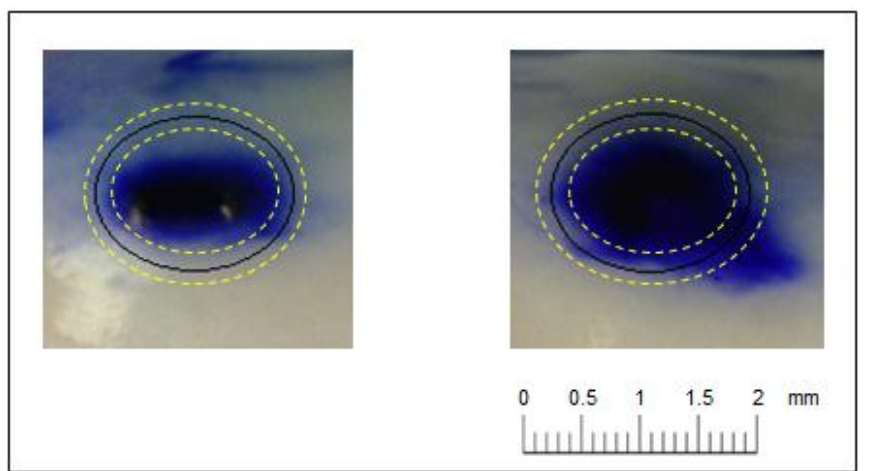

C
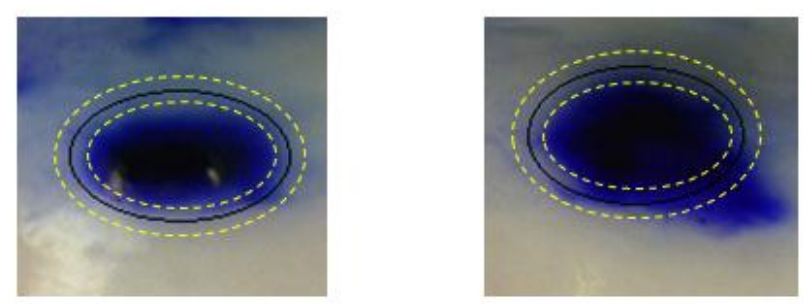

d

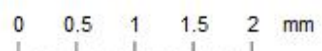

Fig. 4 Ex vivo microdialysis images, superimposed with circles corresponding to the $r_{\text {TVImax }}$. (a) The $r_{\text {TVImax }}$ according to the regression model (black line) and its associated 95\% prediction interval (red lines). (b) - (d) The $\mathrm{r}_{\mathrm{x}}$ and $\mathrm{r}_{\mathrm{y}}$ when using anisotropic vales of $\lambda$, presented with mean value (black line) and standard deviation (yellow lines). $D=4.2 \cdot 10^{-6} \mathrm{~cm}^{2} / \mathrm{s}, k \sim \operatorname{Re}\left(0.003,0.01\right.$ ). (b) $\lambda_{x}=1.51, \lambda_{y}=1.59$ (c) $\lambda_{x}=1.47, \lambda_{y}=1.69$ (d) $\lambda_{x}=$ $1.44, \lambda_{y}=1.95$. 


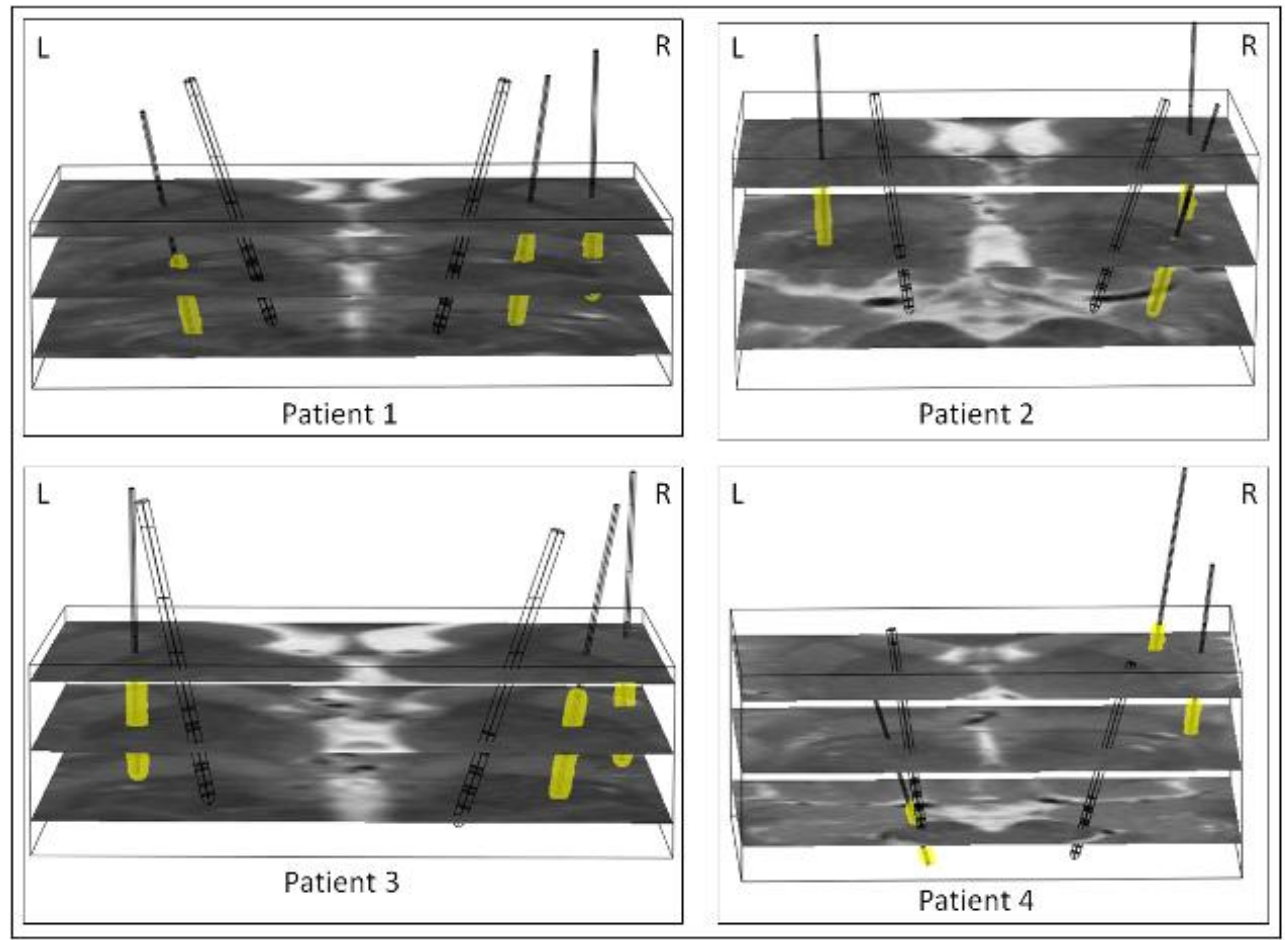

a
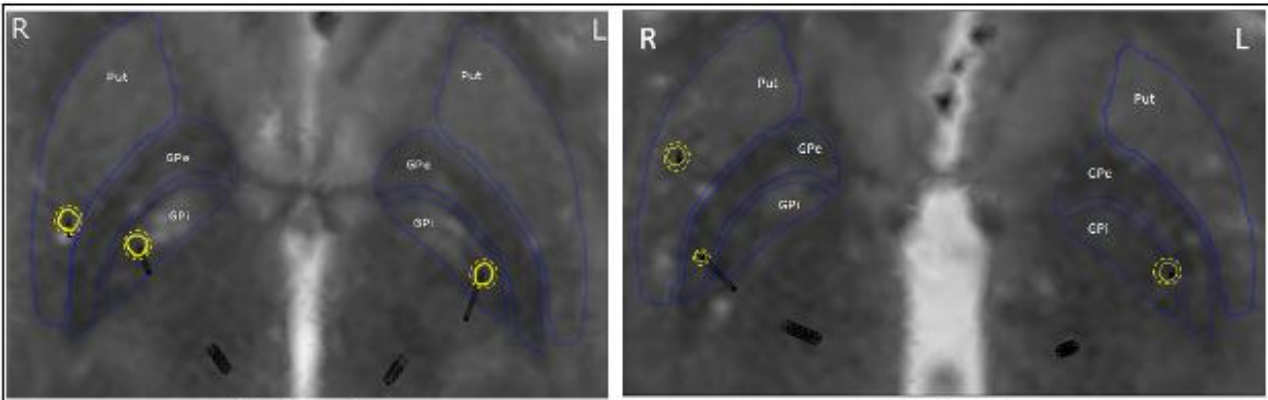

Patient 1

Patient 2
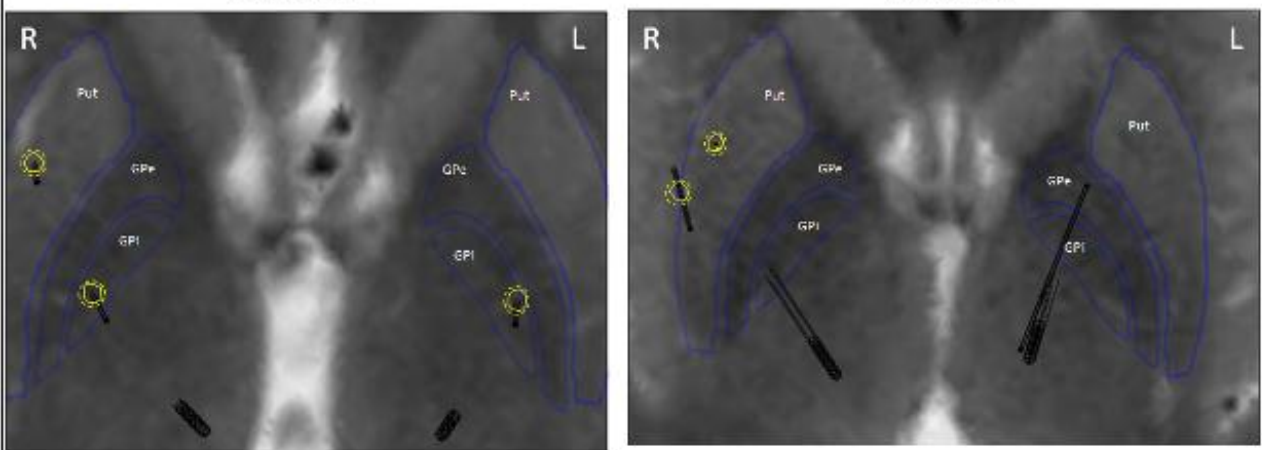

Patient 3

Lัس

Patient 4

b

Fig.5 Visualization of the structure-specific simulations, including the defined $\mathrm{TVI}_{\max }$. Models of the DBS electrodes are included. (a) Spatial overview of the $\mathrm{TVI}_{\max }$ (yellow) for each catheter in relation to patient

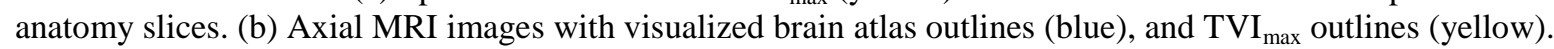
The upper limit of the $95 \%$ prediction interval has been added. 


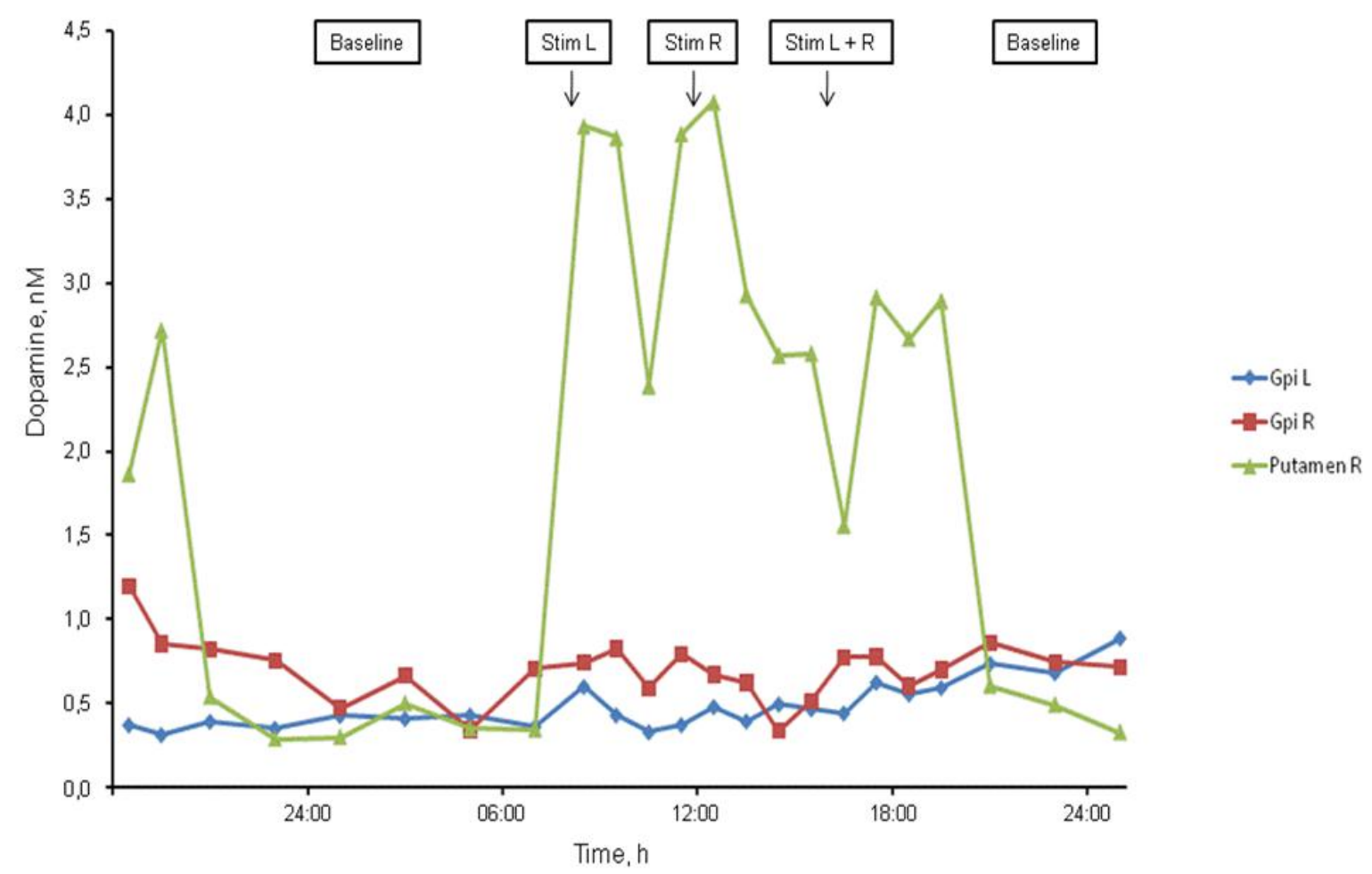

Fig. 6 Microdialysis data for dopamine for Patient 1, during the first 30 hours of the DBS stimulation protocol. Following DBS, an increasing dopamine concentration is seen for one catheter only, showing that there are differences in the biochemical signaling between the targets. 